\title{
The progressing research of traditional Chinese medicine treatment of
}

\section{insomnia}

\author{
Liu Jing ${ }^{1}$, a \\ ${ }^{1}$ Shandong University of Traditional Chinese Medicine, china \\ a793563654@qq.com
}

\section{Keywords: traditional Chinese medicine insomnia}

\begin{abstract}
With the rapid development of society and the increase of the pressure of competition. Insomnia patients increased year by year. Recently, traditional Chinese medicine has made obvious progress in treating insomnia and plays more and more important role in treating insomnia. Traditional Chinese medicine syndrome differentiation and treatment and the characteristics of the multiple targets of action of traditional Chinese medicine compound, make traditional Chinese medicine in the treatment of insomnia have stable curative effect, and the obvious advantages like small side effect and diverse methods. This paper reviews the experimental research and clinical progress of Chinese medicine treatment of insomnia. Patients with insomnia, usually refers to trouble falling asleep, sleep quality decline, total sleep time reduced, the symptom such as wake up at the same time accompanied by daytime dysfunction. it was called cannot sleep ,must not fall down in traditional Chinese medicine. Treatment of insomnia has a history of thousands of years of traditional Chinese medicine, Chinese medicine theory in the books huangdi neijing have records of "stomach lie unsettling". At present, modern medicine in the clinical commonly used sedative hypnotic drugs in the treatment of insomnia, but very prone to resistance and withdrawal reaction, and the blood, cardiovascular, respiratory, produce different degree of adverse reactions, the curative effect of Chinese medicine in the treatment of insomnia showed stable, and the advantages of small side effects, this article summarizes the experimental research and clinical development progress of Chinese medicine treatment of insomnia.
\end{abstract}

\section{Introduction}

diet not festival, aging of several factors such as long illness, usually by imbalance of Yin and Yang, Yang does not enter the Yin, the camp who estrangement and internal imbalances (huangdi neijing) for the pathogenesis and play. Zhao Shaohua [1] don't think of modern first consideration should be insomnia causes, liver for onset of heart for the spread of disease, should pay attention to in the process of treatment of insomnia regulate viscera of heart and liver, adjustment function, make the viscera due to balance of Yin and Yang. Zhang [2] that eating section is the main cause of insomnia, diet section does not affect the function of the spleen and stomach, lead to transport department, the phlegm dampness, and cause insomnia. "Blood exhausted sinar grace [3] said, blood deficiency", "for the disease of depression", "stomach hysteresis heat" is the main factor lead to insomnia, three factors influence each other, make insomnia worse. Xue-wen zhou [4] thinks that the pathogenesis of insomnia is characterized by imbalance of Yin and Yang, the disease of heart and is associated with liver spleen and kidney. Yang in Yin under normal condition, the coordination of Yin and Yang, people can sleep, if blood deficiency, Yin from Yang, Yang excessiveness can't into the shade, or evil disturbed mind, can be caused by imbalance of Yin and Yang to wakefulness. 
Tang dynasty [5] think TCM traditional "differentiation" from the heart often cannot obtain satisfactory curative effect, think of insomnia patients with anxiety state, is closely related to modern disease, thus puts forward the treatment principle of "from liver differentiation".

\section{Chinese medicine decoction}

Traditional Chinese medicine medicinal broth in treatment of insomnia is on the basis of the theory of traditional Chinese medicine syndrome differentiation and treatment. Li ling [6] and so on in zizyphus jujube kernel soup in the treatment of insomnia, according to the evaluation criteria, the clinical total effective rate is $94.20 \%$, and diazepam group total effective rate was $82.80 \%$, two groups of curative effects were significant different. Xue Guang group $(\mathrm{P}<0.05)$ [7] observe 116 cases of insomnia patients, such as the control group taking estazolam (LMG, before sleeping, 1 days), observe the 56 cases, the total effective rate was 73. 21\%. Treatment group taking spleen-invigorating tonga subtraction, observation of 60 cases, the total effective rate was 83. 34\%. After statistics processing, spleen-invigorating tonga subtraction estazolam is obviously better than the clinical curative effect $(\mathrm{P}<0.05)$, and no obvious adverse reactions. Cheap running [8], etc will be insomnia patients with random number table method were randomly divided into treatment group and control group 30 cases, control group capsule oral sleeping in the park, to resolve depression yangxue anshen decoction oral treatment group, for 4 weeks, the curative effect is similar between the two groups have significant differences. $(\mathrm{P}<0.05)$ Wang Lingshu [9] radix bupleuri plus keel oyster soup to treat insomnia, according to the curative effect of evaluation criteria, the total effective rate was $80.00 \%$ after 4 weeks, compared with the control group take sweet dream capsule, the curative effect is similar between the two groups have significant differences. $(\mathrm{P}<0.05)$. In one hundred thousand [10] observing 60 patients with insomnia, 30 cases were randomly divided into control group, treatment group 30 cases. Treatment group is given priority to with Dan mast joint huanglian wendan tang xiaoyao powder, and with the disease to add and subtract, according to the curative effect of evaluation criteria, the total effective rate is $89.47 \%$, and taking estazolam (2 mg each time, before sleeping, take, 1 days) compared to the control group, two groups of curative effects have significant difference. $(\mathrm{P}<0.05)$. Anyhow, involved in the treatment of insomnia in the formulas, the high frequency with the formulas zizyphus jujube kernel soup, radix bupleuri add keel oyster soup, soup of spleen-invigorating, nourishing blood anshen decoction, radix bupleuri add keel oyster soup, huanglian wendan tang, etc.

\section{Acupuncture treatment}

Luo fei [11] observe 82 cases of insomnia patients, 39 cases in treatment group, control group 43 cases. Acupuncture treatment group selected point: the main point is chosen, too creek, four god astute. Control group: taking estazolam alone $2 \mathrm{mg}$, before sleeping, 1 days, continuous treatment after 4 weeks of treatment group total effective rate was $90.69 \%$, control group total effective rate was $71.79 \%$, clinical curative effect of acupuncture is superior to take estazolam alone $(\mathrm{P}<0.05)$ and no obvious adverse reactions. Hong [12] observe 66 cases of insomnia patients, the treatment group and control group with 33 cases. Acupuncture treatment group main point chosen door hole, the hole, four god our acupuncture point, within the cave, and combining the insomnia patients with specific types to choose other points as deputy. Patients with phlegm heat in one type choose hong leong hole and Chambers hole as vice hole; Yin deficiency type fire patients choose too creek hole and lr 3 hole as vice hole; According to the curative effect of evaluation criteria, the treatment group total effective rate was $97.0 \%$, compared with the control group taking alprazolam, two groups of 
curative effects were significant different $(\mathrm{P}<0.05)$. Fan Xiaoyan [13] insomnia patients using the random number table method were randomly divided into treatment group (47 cases, control group, 42 cases. Acupuncture treatment group main point will choose best hole, four god astute hole, cave door god, according to the acupuncture point, shen mai acupuncture point, sleeping, and connecting with the concrete type choice selected point in patients with insomnia. According to the curative effect of evaluation criteria, the treatment group total effective rate was 93.60\%, and compared with the control group taking estazolam, two groups of curative effects were significant. $(\mathrm{P}<0.05)$

\section{Massage therapy}

Chen Zhichang [14] observe 76 cases of insomnia patients, among them, 38 cases in the control group and treatment group. To treatment group is given priority to with massage therapy, according to the curative effect evaluation standard, total effective rate up to $97.40 \%$. Control group patients give routine drug treatment, drug choice lorazepam, according to the proposed the curative effect of evaluation criteria, the total effective rate of $78.90 \%$. Control group total effective rate significantly lower than the total effective rate in treatment group, two groups of curative effects were significant. ( $\mathrm{P}<0.05$ ) PangJun [15] is made of massage foot shaoyang bile after treatment of 160 cases of insomnia, oral estazolam drug group (1 / d) $1 \mathrm{mg}$ each time, bedtime, treatment after 30 times in a row, the control group total effective rate in the therapeutic effect evaluation standard of quasi significantly lower than the treatment group total effective rate, two groups of curative effects were significant. ( $\mathrm{P}<0.05)$ hong-liang tang $[16]$ observe 80 cases of insomnia patients, the treatment group and control group with 40 cases. Control group oral estazolam (1 / d) 1 mg each time, bedtime treatment group with Yang white, cheng ling, our god, and the rate of the valley, camp, head Lin chi, wind pool and so on acupuncture points; Two groups of treatment 1 times a day, 1 months after continuous treatment, the treatment group and control group total effective rate were 92. $1 \%$ and 84 . $2 \%$, two groups of curative effects were significant. $(\mathrm{P}<0.05)$

\section{Acupoint sticking therapy}

Xiang-dong wu [17] observe 80 cases of insomnia patients, the treatment group 40 cases, control group 40 cases. Treatment group on the basis of conventional treatment of traditional Chinese medicine acupoint sticking therapy on both sides of three vaginal intercourse and yongquan, and according to the sea. Control group: taking estazolam alone $2 \mathrm{mg}$, before sleeping, 1 days, continuous treatment of 8 weeks after treatment group total effective rate was $93.30 \%$, control group total effective rate was $82.20 \%$, Chinese medicine acupuncture applied clinical curative effect is better than taking estazolam alone $(\mathrm{P}<0.05)$ and no obvious adverse reactions. Shi Huawei [18] with acupoint sticking therapy of traditional Chinese medicine insomnia (fu patient 71 cases, 60 patients with san-jiu), according to the PSQI score curative effect evaluation standard, sleep after acupoint sticking therapy, the total effective rate 85.5\%, patients with PSQI were significantly improved (PG < 0.01).

\section{Comprehensive therapy}

The clinical treatment of insomnia, the application of several methods are often combined treatment of traditional Chinese medicine in addition to the traditional Chinese medicine (TCM), the common acupuncture, massage, and cupping, needle navel, cans and other methods. Pan Xianming [19] the 122 cases of patients with insomnia were randomly assigned to treatment group by random number table method in 62 cases of 60 cases with control group, treatment group for 
electric acupuncture group, the treatment group as the routine acupuncture group, both groups take god door, four chung, wind pool hole is given priority to, such as 30 days' control group total effective rate and the treatment group total effective rate were $90.32 \%$ and $75.00 \%$ respectively, the results in the control group total effective rate significantly lower in treatment group total effective rate $(\mathrm{P}<0.05)$. Liu Yu [20] will observe 164 cases of insomnia patients, the treatment group 82 cases and control group in 8 cases, treatment group for umbilical needle treatment group and control group for regular foot three mile, four god astute, door god acupuncture group, the results showed that umbilical needle treatment group compared with control group of PSQI score were significantly improved.

\section{Conclusion}

Traditional Chinese medicine in the treatment of insomnia in clinical practice, give full play to the characteristics of evidence-based medicine of traditional Chinese medicine, has obvious advantages. Clinical treatment of insomnia can be various, multi-level, can choose Chinese medicine medicinal broth, cupping, acupuncture, massage and other methods. Hope that the future of traditional Chinese medicine combined with modern medical research methods, giving full play to the advantages of TCM syndrome differentiation, improve the quality of sleep, so as to benefit the human beings.

\section{Reference}

[1] Liang Limin. Zhao Shaohua chief physician experience insomnia analyses [J]. Modern combine traditional Chinese and western medicine journal, 2017, (01) : 98-100.

[2] zhang. 57 cases of clinical observation comprehensive therapy to treat sleep disorders [J]. China's health industry, 2012 (28).

Ann. [3] fan province sinar yi, director of the physician's clinical experience in the treatment of insomnia summary [D]. University of Chinese medicine in gansu province, 2016.

[4] Hou Yan Chen Min. Xue-wen zhou experience insomnia treatment [J]. Journal of liaoning traditional Chinese medicine journal, 2015 (6) : 1196-1198.

[5] Liang Jing, li ning, lu to static, etc. Professor tang dynasty treat insomnia clinical experience [J]. The light of traditional Chinese medicine, 2016, 31 (8) : 1082-1084.

[6] li ling. The curative effect of decoction of semen ziziphi spinosae (zs) 35 cases of insomnia [J]. Journal of clinical medicine literature electron, 2015 (4) : 641-641.

[7] Xue Guang. Spleen-invigorating tonga subtraction type glove two virtual 60 cases of primary insomnia curative effect observation [J]. National physician BBS, 2016 (2) : 28 and 29.

[8] inexpensive running. Tian-wen zhang treating insomnia the academic experience of professor [C] / / at the Chinese academy of traditional Chinese and western medicine combined with health and rehabilitation medicine professional committee meeting and eighth academic seminar. 2013.

[9] Wang Lingshu, li yiyun, cheung chi-kong. Radix bupleuri add keel oyster soup treating primary insomnia clinical observation [J]. Journal of shaanxi traditional Chinese medicine, 2016, 37 (9) : 1170-1171.

[10], one hundred thousand. Dan Zhi xiaoyao powder and huanglian WenDan tonga subtraction treatment of insomnia clinical observation [J]. Journal of traditional Chinese medicine clinical research, 2015 (7) : 87-88.

[11] luo fei. Clinical observation of acupuncture treatment insomnia [J]. Chinese folk therapy, 2017. (01) : 12-13. 
[12] hong. The clinical effect of the treatment of acupuncture treatment for insomnia [J]. Journal of clinical medicine literature electron, 2016, 3 (15) : 3047-3047.

[13] Fan Xiaoyan. The clinical effect of the treatment of acupuncture treatment for insomnia [J]. Journal of clinical medical research and practice, 2016, 1 (24).

[14] Chen Zhichang, jian-wei xing, li ying. The massage treatment of insomnia, 38 cases of recent clinical results analysis [J]. The world's latest medical information abstract: continuous electronic journal, 2015, 15 (22) : 10-11.

[15] PangJun Hu Yundan, Chen, etc. Pivot through massage therapy fatigue sex insomnia theoretical exploration [C] / / the 12th national academic conference and massage massage modulation of sub-health classes on clinical application and research progress. 2011.

[16] hong-liang tang, Chen, PangJun, etc. Pivot the massage treatment of insomnia: a randomized controlled study [J]. Chinese acupuncture and moxibustion, 2015, 35 (8) : 816-818.

[17] xiang-dong wu. The clinical effect of Chinese medicine acupoint sticking therapy of insomnia explore [J]. Journal of contemporary Chinese medicine, 2013, 20 (10) : 106-106.

[18] Shi Huawei XingJia, jia-lin wang, etc. Solar term acupoint sticking on the quality of the patients with insomnia sleep [J]. Journal of Chinese medicine, 2016, 31 (1) : 135-137.

[19] Pan Xianming Zhang Li, Yang Yang, etc. Electric acupuncture treatment of insomnia curative effect observation [J]. Journal of traditional Chinese and western medicine combined with cardiovascular disease the electronic magazine, 2016, 4 (12) : 51-52.

[20] Liu Yu. Umbilical needle treatment of insomnia clinical observation [J]. Journal of liaoning university of traditional Chinese medicine, 2009 (11) : 149-151. 